\title{
Sujet lecteur et lecture littéraire : quelles modélisations pour quels enjeux?
}

Jean-Louis Dufays

\section{OpenEdition}

\section{Journals}

\section{Édition électronique}

URL : http://journals.openedition.org/recherchestravaux/666

DOI : 10.4000/recherchestravaux.666

ISSN : 1969-6434

Éditeur

UGA Éditions/Université Grenoble Alpes

\section{Édition imprimée}

Date de publication : 31 décembre 2013

Pagination : 77-88

ISBN : 978-2-84310-267-7

ISSN : 0151-1874

Référence électronique

Jean-Louis Dufays, "Sujet lecteur et lecture littéraire : quelles modélisations pour quels enjeux? », Recherches \& Travaux [En ligne], 83 | 2013, mis en ligne le 01 juillet 2015, consulté le 10 décembre 2020. URL : http://journals.openedition.org/recherchestravaux/666 ; DOI : https://doi.org/10.4000/ recherchestravaux.666 


\section{Sujet lecteur et lecture littéraire : quelles modélisations pour quels enjeux?}

\section{Posons le problème}

Quatre publications récentes sont à l'origine de la présente réflexion.

La première est l'analyse de Brigitte Louichon ${ }^{\mathrm{I}}$, qui, après avoir mené l'enquête sur les définitions actuelles de la «lecture littéraire», constate la coexistence de deux tendances contradictoires : d'un côté, une conception large, qui voit cette activité comme «une lecture centrée sur le lecteur, menée dans une communauté interprétative au moyen de la parole échangée»; de l'autre, une conception plus précise, qui la définit comme un va-et-vient dialectique entre des postures de lecture complémentaires. Louichon constate cependant que la tension entre ces deux approches semble échapper à la plupart des acteurs. En effet, si ceux-ci se réfèrent très largement aux travaux qui définissent la lecture littéraire comme va-et-vient, dans leurs écrits, «l'usage formel de la référence [à ces travaux] apparaît plus comme une soumission à la rhétorique de la communication que comme un véritable outillage théorique ${ }^{2} »$. Dans les faits, c'est dès lors le sens large du lexème «lecture littéraire » qui domine, mais sans être clairement assumé comme tel.

La deuxième publication est celle des actes du colloque «Le Texte du lecteur» qui s'est tenu à Toulouse en $2008^{3}$. Dans l'introduction du premier

I. B. Louichon, "La lecture littéraire est-elle un concept didactique?», dans R. Goigoux et M.-C. Pollet (dir.), Approches didactiques de la lecture, Namur, AIRDF - Presses universitaires de Namur, 20II, p. 195-2I6.

2. Ibid., p. 207.

3. C. Mazauric, M.-J. Fourtanier, et G. Langlade (dir.), Le Texte du lecteur, Bruxelles, Peter Lang, coll. "ThéoCrit", 20 II. 
volume, ses coordinateurs articulent la notion de lecture littéraire à celle de "sujet lecteur", qu'ils présentent comme son complément naturel. Cette articulation est cependant contredite dans une troisième publication, signée par Jean-Louis Dumortier ${ }^{4}$, qui hiérarchise quant à lui lecture littéraire et sujet lecteur et propose de renoncer à la première au profit du second.

La quatrième publication est la traduction française du livre de Stanley Fish, Quand lire c'est faires, qui traite centralement de la place du sujet dans la lecture.

La simple évocation de ces ouvrages suffit à montrer combien la réflexion théorique autour de la lecture et de son enseignement continue aujourd'hui à faire débat. Étant moi-même engagé dans ce débat depuis plus de vingt ans, je voudrais essayer ici d'en clarifier les termes et d'en mesurer les intérêts. Mon souci, ce faisant, est de poser un regard métacritique sur les enjeux et sur les difficultés didactiques liés aux notions de lecture littéraire et de sujet lecteur, ainsi que sur les relations qui unissent ces notions.

Quatre étapes scanderont ma réflexion. Je ferai d'abord le point sur la notion de lecture littéraire, dont j'interrogerai la nature, les enjeux et les limites. Je m'intéresserai ensuite aux contours du sujet lecteur, ce qui me permettra, dans un troisième temps, d'étudier les relations possibles entre les deux notions. J'évoquerai enfin les pistes d'action qu'il me semble permis d'envisager pour approfondir l'exploitation féconde de ces deux notions dans les recherches en didactique.

\section{Le point sur la lecture littéraire}

\section{Trois orientations}

Comme j'ai déjà eu l'occasion de l'écrire ${ }^{6}$, depuis qu'elle est devenue une notion heuristique pour la recherche en littérature et en didactique, la lecture littéraire a été étudiée selon trois orientations distinctes.

La première privilégie la distanciation critique : la lecture littéraire y est conçue comme l'activité d'un lecteur exigeant, désireux de vivre une expérience esthétique ouverte sur l'écart, le symbole, la polysémie, respectueuse des «droits du texte» et de certaines règles interprétatives communes. C'est cette conception

4. J.-L. Dumortier, Devenir un professionnel de l'enseignement du français (sans perdre le goût de l'enseigner), Namur, Presses universitaires de Namur, coll. "Dyptique», $201 \mathrm{.}$

5. S. Fish, Quand lire c'est faire. L'Autorité des communautés interprétatives, Paris, Les Prairies ordinaires, 2007.

6. J.-L. Dufays, L. Gemenne et D. Ledur, Pour une lecture littéraire. Histoire, théories, pistes pour la classe, Bruxelles, De Boeck - Duculot, coll. «Savoirs en pratique», 2005. 
que préconisent - ou préconisaient, car certaines positions ont bougé au fil du temps - des auteurs comme Marghescou ${ }^{7}$, Gervais $^{8}$, Tauveron ${ }^{9}$ ou Rouxel ${ }^{\text {Io }}$.

La deuxième approche privilégie à l'inverse la participation psychoaffective, la lecture libre, l'appropriation personnelle, c'est-à-dire l'activité du lecteur "ordinaire», qui donne la priorité aux émotions, aux processus d'identification et de reconfiguration subjective. Cette conception trouve ses meilleurs avocats chez de Certeau", qui conçoit la "lecture-braconnage» comme une des modalités essentielles de "l'invention du quotidien", chez Poslaniec ${ }^{12}$, qui fait de la surprise l'expérience clé de la lecture littéraire, chez Daunay ${ }^{13}$, qui s'intéresse aux processus de réception ordinaires comme celui de la paraphrase, mais aussi chez Bayard ${ }^{14}$, dont les divers ouvrages soulignent la part inaliénable d'autonomie dont dispose tout lecteur.

La troisième approche enfin saisit la lecture littéraire dans le va-et-vient entre les deux approches précitées : elle conçoit cette activité comme celle d'un lecteur " complet", qui joue sur les deux tableaux à la fois, celui de la raison et celui des passions, celui de la construction du sens et celui de l'illusion référentielle, celui de la compréhension et celui de la progression. Comme on le sait, cette conception a été théorisée par Picard ${ }^{15}$, avant d'être reformulée, précisée et prolongée didactiquement par mes propres travaux en $1996\left(2^{\mathrm{e}}\right.$ édition : 2005) et par ceux de quelques collègues, en particulier Annie Rouxel à partir des années 2000 ${ }^{16}$.

\section{Quatre mises au point}

Si, comme le constate Brigitte Louichon, la troisième approche est aujourd'hui le plus souvent citée en tant que référence d'autorité, il faut bien

7. M. Marghescou, Le Concept de littérarité. Critique de la métalittérature [Mouton, 1974], Kimé, 2009.

8. B. Gervais, À l'écoute de la lecture, Montréal, VLB éditeur, 1993.

9. C. Tauveron, «Comprendre et interpréter le littéraire à l'école : du texte réticent au texte proliférant ", Repères, n ${ }^{\circ}$ 19, 1999, p. 9-38.

Io. A. Rouxel, Enseigner la lecture littéraire, Rennes, Presses universitaires de Rennes, coll. "Didact Français», 1996.

II. M. de Certeau, L'Invention du quotidien. I. Arts de faire, Paris, Gallimard, coll. «Folio essais ", I980.

I2. Ch. Poslaniec, De la Lecture à la littérature, Paris, Sorbier, 1992; Vous avez dit «littérature»?, Paris, Hachette, 2002.

I3. B. Daunay "La lecture littéraire : les risques d'une mystification ", Recherches, nº 30, I999, p. 29-59; éloge de la paraphrase, Vincennes, Presses universitaires de Vincennes, 2002.

I4. P. Bayard, Comment parler des livres que l'on n’a pas lus, Paris, Minuit, coll. «Critique», 2007.

I5. M. Picard, La Lecture comme jeu, Paris, Minuit, coll. «Critique», I986.

16. A. Rouxel et G. Langlade (dir.), Le Sujet lecteur. Lecture subjective et enseignement de la littérature, Rennes, Presses universitaires de Rennes, 2004. 
constater que cela n'empêche pas la notion de lecture littéraire de rester, dans bon nombre d'usages, l'objet d'un flou notionnel assez persistant. En l'occurrence, quatre sources d'imprécisions, qui me semblent autant de causes de malentendus, peuvent être pointées.

En premier lieu, sur quels éléments porte au juste le va-et-vient de la lecture littéraire? Le mouvement dialectique qui est le plus souvent évoqué concerne des modalités opposées de construction du sens : régime de lecture ordinaire et régime de lecture littéraire d'après Marghescou, réception quasi pragmatique et réception pseudo-référentielle ${ }^{17}$, lu et lectant ${ }^{18}$, lisant et lectant ${ }^{19}$, lecture ancrée pragmatiquement et lecture ancrée esthétiquement ${ }^{20}$, régime de la progression et régime de la compréhension ${ }^{21}$, ou encore participation et distanciation ${ }^{22}$. Picard cependant allait plus loin en parlant d'un va-et-vient entre des modalités d'évaluation opposées, qu'il résumait comme suit : «Subversion dans la conformité, élection du sens dans la polysémie, modélisation par une expérience de réalité fictive ${ }^{23}$.» J'ai montré quant à moi que ce triple jeu mettait chaque fois en tension un critère axiologique qu'on peut qualifier de "classique» (la conformité, le sens, la modélisation) et un autre qu'on peut qualifier de "moderne» (la subversion, la polysémie, l'expérience de réalité fictive), et qu'il paraissait pertinent de compléter le modèle en considérant que les valeurs peuvent faire l'objet d'au moins trois autres mouvements dialectiques : celui qui articule les valeurs de l'émotion à celles de la raison, celui qui articule les valeurs liées à la fonction poétique à celles qui relèvent de la fonction référentielle, et celui qui articule les valeurs de l'éthique à celles de sa transgression ${ }^{24}$.

Autrement dit, si l'on suit la définition de Picard, la lecture littéraire ne consiste pas seulement à osciller entre des postures de réception différentes; elle s'attache, plus avant, à soumettre le texte à des évaluations contradictoires et à trouver dans chacune d'elles des motifs de satisfaction. Lire littérairement, selon cette conception, c'est faire de sa lecture un jugement de valeur « riche», qui s'astreint à mobiliser une diversité de critères et d'axiologies.

17. K. Stierle, «Réception et fiction», Poétique, n 39, 1979, p. 299-320.

18. M. Picard, La Lecture comme jeu, ouvr. cité.

19. V. Jouve, La Lecture, Paris, Hachette, 1993.

20. B. Lahire "L'inégalité devant la culture écrite scolaire : le cas de l'“expression écrite" à l'école primaire", Sociétés contemporaines, $\mathrm{n}^{\circ} \mathrm{II}, \mathrm{I} 992$.

21. B. Gervais, À l'Écoute de la lecture, ouvr. cité.

22. J.-L. Dufays, Stéréotype et lecture. Essai sur la réception littéraire [Mardaga, 1994], Berne, Peter Lang, coll. "ThéoCrit" », 2010.

23. M. Picard, La Lecture comme jeu, ouvr. cité, p. 266.

24. J.-L. Dufays, "Lire, c'est aussi évaluer. Autopsie des modes de jugement à l'œuvre dans diverses situations de lecture", Études de linguistique appliquée, $\mathrm{n}^{\circ}$ II9, 2000, p. 277-290. 
En deuxième lieu, l'expression «va-et-vient dialectique» est parfois, en ellemême, l'objet d'interprétations diverses. Certains chercheurs imaginent en effet qu'elle désigne une oscillation permanente entre deux postures "pures", c'est-àdire un mouvement spectaculaire qui ferait passer le lecteur constamment d'un extrême (par exemple l'immersion référentielle «totale») à l'autre (par exemple l'analyse critique systématique). Le discours que j'ai, pour ma part, toujours essayé de tenir à ce propos se veut beaucoup moins radical : il insiste plutôt sur le fait que les postures concernées se situent sur un continuum, et qu'elles sont donc rarement dans un rapport d'incompatibilité. Pour le dire autrement, la grande majorité des lectures distanciées comportent une part de participation, et inversement. Parler de va-et-vient dialectique signifie simplement qu'au cours de sa lecture, le lecteur mobilise tour à tour des accentuations différentes, en étant tantôt plus ancré dans l'illusion référentielle, tantôt plus attaché à la réflexion critique... sans avoir besoin pour autant de jamais radicaliser l'une ou l'autre attitude.

Une troisième difficulté liée à la notion de lecture littéraire tient au caractère prioritairement propositionnel de son usage : elle a davantage été construite pour servir de modèle aux formateurs et pour inspirer l'action des enseignants et des élèves que pour aider à décrire les lectures réelles. En soi, ce n’est pas un problème, mais il en résulte un malentendu chez certains chercheurs, qui voulant faire de ce modèle formatif un outil descriptif, s'étonnent de ne pas trouver dans les pratiques spontanées des enseignants ou des élèves des manifestations massives du va-et-vient dialectique. Ce malentendu peut certes être dépassé si l'on adhère à la conception souple du va-et-vient évoquée dans le paragraphe précédent : à cette condition, la grande majorité des réceptions d'œuvres littéraires peuvent être décrites comme activant une tension dialectique plus ou moins marquée. Il faut toutefois insister sur le fait que cette tension intéresse la didactique davantage comme pratique à promouvoir que comme compétence déjà acquise.

Enfin, un malentendu persistant concerne la nature «littéraire» de l'activité. Selon Dumortier ${ }^{25}$, l'usage didactique de la notion correspondrait au « rassemblement de transpositions scolaires des lectures savantes». Pourtant, quand on examine la majorité des travaux existants - les miens comme ceux de mes collègues -, la rupture paraitt nette entre les usages savants de la lecture littéraire et sa transposition didactique, qui, dans les faits, apparaît bien davantage comme "ascendante" (fondée sur l'observation des pratiques) que comme «descendante» (dérivée de modèles abstraits). En effet, parler de va-et-vient entre lu/lisant et lectant ou entre participation et distanciation, ce n'est pas

25. J.-L. Dumortier, Devenir un professionnel de l'enseignement du français..., ouvr. cité, p. I03. 
projeter sur les lectures scolaires une pratique de spécialistes : c'est seulement proposer aux enseignants et aux élèves d'accentuer à des fins didactiques un mouvement qui serait déjà consubstantiel à toute lecture. Si l'un des pôles de la dialectique se situe du côté de la distanciation, laquelle, dans certains cas, peut recourir à des outils de lecture "savante», sa mobilisation n'est nullement l'apanage des spécialistes : elle fait partie de la lecture «ordinaire». Le fait que la lecture littéraire fonctionne comme un modèle didactique ne l'assimile donc en rien à cette "lecture savante» dont Dumortier se méfie à juste titre.

\section{Les enjeux du va-et-vient}

Ces mises au point étant faites, il m'importe de rappeler l'enjeu de cette conception dialectique de la lecture. Par son caractère intégrateur, celle-ci permet en effet de promouvoir une action didactique équilibrée face aux deux dérives que sont d'un côté la conception textualiste et ses avatars (qui tendent à ignorer les variations de la lecture réelle pour la conformer aux formats d'une lecture "modèle») et de l'autre la conception subjectiviste, caractérisée par son absence de limites et, partant, son caractère inenseignable et inévaluable. En mobilisant tour à tour deux modalités de lecture et deux axiologies apparemment opposées, l'enseignant permet à l'élève lecteur à la fois d'épanouir sa part de liberté interprétative et évaluative et de l'inscrire dans les balises d'une activité collective tendue vers la quête d'un sens commun et de valeurs partagées, ce qui correspond bien, me semble-t-il, à la double mission de l'école en matière de formation à la lecture.

À cela s'ajoute l'hypothèse que le va-et-vient constituerait la tendance « déjà là " de toute lecture, selon des degrés divers : penser la lecture littéraire comme un modèle d'action à promouvoir n'empêche pas de percevoir sa continuité avec les autres formes de lecture, dont elle se distinguerait seulement par son intensité et/ou sa réflexivité. L'idée est ici de considérer que toute lecture comporte, peu ou prou, une dimension dialectique, que la notion de lecture littéraire rend visible et contribue à accentuer.

Concluons provisoirement cette première analyse en constatant qu'au-delà de son usage «large», qui lui permet de désigner les pratiques de lecture quelconques échangées dans le cadre de la classe, la notion de lecture littéraire gagne à servir, beaucoup plus précisément, de modèle et d'outil pour promouvoir une conception dialectique de la lecture, qui rejoint un enjeu didactique majeur. Certes, chacun reste libre de se contenter de la seule acception large, mais on peut douter que celle-ci aide à faire progresser significativement les pratiques. 


\section{Le point sur le sujet lecteur}

La notion de sujet lecteur, quant à elle, est apparue pour la première fois dans le champ de la didactique à l'occasion du colloque éponyme qui s'est tenu à Rennes en janvier 2004. En introduisant ce colloque, Annie Rouxel a présenté la mise au premier plan du «sujet lecteur » comme une réaction contre le «lecteur modèle» d'Eco ${ }^{26}$. La notion a ensuite fait l'objet d'une définition plus avancée à Toulouse, en octobre 2008 , à l'occasion du colloque «Le texte du lecteur ${ }^{27}$ » qui s'intéressait aux divers types d'appropriation des textes.

L'évolution des références invoquées pour fonder la notion est intéressante à observer. En 2004, dans les propos de Rouxel et de Langlade, c'était surtout Picard qui faisait figure d'autorité, ce qui ancrait clairement le sujet lecteur dans la théorie de la lecture littéraire; mais dans les propos de Mazauric, Fourtanier et Langlade $^{28}$, il est plutôt question de Bellemin-Noël ${ }^{29}$, de Bayard, de Petit ${ }^{30}$, de de Certeau... et donc de fondements théoriques qui promeuvent une conception "émancipatrice» de la subjectivité, où l'expression libre du sujet apparaît comme une fin en soi plutôt que comme une phase dans un processus plus global. Les auteurs de 201 masquent cependant cette évolution en affirmant que déjà ceux de 2004 avaient souligné «l'importance de la subjectivité, de la singularité, voire de la transgressivité des lecteurs empiriques dans la lecture littéraire, ordinaire et critique, naïve et experte, privée et scolaire ${ }^{3 \mathrm{I}} »$.

Dans ces conditions, quelle définition du sujet lecteur faut-il retenir? Une certitude est que ses promoteurs s'accordent à assimiler celui-ci au pôle empirique des théories de la réception, c'est-à-dire au lecteur effectif, considéré dans toutes ses dimensions. Gérard Langlade a en outre caractérisé le sujet lecteur par sa triple activité de "fictionalisation", qu’il nomme «la vision du monde», «l'imagination» et «l'axiologisation $»^{32}$.

26. U. Eco, Lector in fabula. La coopération interprétative dans les textes narratifs, Paris, Grasset, 1985 .

27. C. Mazauric, M.-J. Fourtanier et G. Langlade (dir.), Textes de lecteurs en formation, Bruxelles, Peter Lang, coll. "ThéoCrit" ", 2oII.

28. G. Langlade et M.-J. Fourtanier, «La question du sujet lecteur en didactique de la lecture littéraire», dans E. Falardeau, C. Fischer, C. Simard, N Sorin (dir.), Les Voies actuelles de la recherche en didactique du français, Québec, Presses de l'université Laval, 2007, p. IOI-I23.

29. J. Bellemin-Noël, Vers l'inconscient du texte, Paris, Presses universitaires de France, coll. «Écriture», I979.

30. M. Petit, Éloge de la lecture. La construction de soi, Paris, Belin, 2002.

31. C. Mazauric, M.-J. Fourtanier et G. Langlade (dir.), Le texte du lecteur, Bruxelles, Peter Lang, coll. "ThéoCrit" ", 20II, p. I9.

32. G. Langlade, "Quelle théorie de la lecture littéraire?» dans J.-L. Dumortier et M. Lebrun (dir.), Une formation littéraire malgré tout, Namur, Presses universitaires de Namur, 2006, 
Pour le reste, quand on lit les textes qui ont été consacrés récemment à ce thème ${ }^{33}$, c'est surtout la diversité de leurs approches qui retient l'attention. En l'occurrence, il me semble que l'on peut y distinguer au moins trois sortes de «sujets lecteurs».

La première correspondrait à la conception normative d'un lecteur subtil, créatif, inspiré, qui, fort de sa culture et de sa finesse, s'insinuerait dans les blancs du texte pour le "co-créer». "Sujet lecteur» se situerait alors assez nettement du côté du lectant et servirait à désigner une posture "savante", accessible à une minorité d'élèves.

La seconde conception serait radicalement émancipatrice : elle renverrait à l'idée d'un lecteur de «jouissance» (Barthes), déconstructeur (de Man), disséminateur (Derrida), transgressif (Bayard)... et donc à une posture qui revendique encore davantage les droits du lecteur face à ceux du texte, mais qui, du coup, peut apparaître comme encore plus «lettrée» et "élitiste» (au sens intellectuel et culturel du terme) que la précédente.

La troisième conception serait non normative : elle correspondrait à l'attitude du lecteur «ordinaire» effectif, considéré dans toutes ses réalisations, notamment celles qui relèvent de la participation affective, ce qui veut dire qu'un tel sujet lecteur pourrait très bien être «naïf» ou «faible». Le sujet lecteur, ici, ce serait simplement le lecteur réel, tel qu'on peut l'observer, y compris lorsqu'il se fourvoie sur des éléments qui relèvent du sens commun, ou à l'inverse, lorsqu'il se soumet strictement aux «droits du texte».

Il me semble que les didacticiens n'ont pas grand intérêt à promouvoir la deuxième conception : il serait paradoxal en effet que, sous couvert de réhabiliter le sujet, le privilège accordé à une conception pointue de la lecture revienne dans les faits à exclure la majorité des élèves du processus ${ }^{34}$.

À l'inverse, la troisième conception présente un enjeu didactique évident, dans la mesure où promouvoir la reconnaissance de l'élève lecteur en tant que sujet «libre» permet de mieux connaître ses fonctionnements effectifs et de remédier à ses difficultés. Une telle conception du sujet lecteur permet aussi

p. 17-20; "L'activité «fictionnalisante» du lecteur », dans B. Louichon et B. Laville (dir.), Les Enseignements de la fiction. Modernités, Bordeaux, Presses universitaires de Bordeaux, 2006, p. I63-177.

33. Je pense ici bien sûr aux deux ouvrages collectifs déjà cités. Je me garderai toutefois d'attribuer chaque conception à des auteurs précis, car, d'une part, les positions à ce propos me paraissent assez mobiles, et d'autre part, je sais qu'il peut être désagréable pour un auteur de se voir étiqueté dans une catégorie forcément réductrice par rapport à la complexité de ses positions.

34. En fait, le risque ici serait exactement le même que celui que Dumortier attribue à la littérature littéraire telle qu'il la comprend. 
de libérer la parole des élèves sur la lecture (comme nous y invitait déjà de Certeau) et de les inciter à donner un sens personnel à leurs lectures.

Il semblerait problématique cependant de limiter le travail en classe à la conception non normative, car l'usage scolaire de la lecture serait alors cantonné à un partage de subjectivités où l'élève n'apprendrait rien, resterait enfermé dans son propre horizon. Or il est aussi membre d'une communauté qui partage certains stéréotypes, certains modes de lecture, et qu'il s'agit de renforcer si l'on veut garantir une pérennité du lien social face au solipsisme d'une individualisation sans borne, ou bornée seulement par la culture mass-médiatique.

Fish nous rappelle d'ailleurs que rien n'est plus social et partagé que les interprétations qui se croient singulières :

Si l'ego est conçu, non comme une entité indépendante mais comme une construction sociale dont les opérations sont délimitées par les systèmes d'intelligibilité qui l'informent, alors les significations qu'il confere au texte ne sont pas les siennes, mais trouvent leur source dans la (ou les) communautés interprétative(s) sur laquelle (ou lesquelles) il repose ${ }^{35}$.

En somme, si elles doivent être prises au sérieux - comme le préconisaient déjà les travaux sur la lecture littéraire -, les lectures subjectives ne constituent qu'une étape de l'apprentissage : elles sont à la fois la base sur laquelle une lecture commune pourra s'élaborer et une occasion pour le sujet de s'approprier le texte en le reliant à sa propre histoire, mais leur didactisation exclusive conduirait à des apories tout aussi contre-productives que celles qui accompagnaient la notion de «lecture modèle».

\section{Quelles relations entre lecture littéraires et sujet lecteur?}

Les notions de lecture littéraire et de sujet lecteur étant ainsi quelque peu clarifiées, il est possible maintenant de revenir sur la question de leur complémentarité.

$\mathrm{Si}$, comme on l'a vu, celle-ci était généralement postulée par leurs promoteurs, elle ne fait pas pour autant l'unanimité, puisque Dumortier attribue aux concepteurs de la lecture littéraire une démarche "offusquant le sujet lecteur [...] au profit du lecteur modèle, au lieu de prendre comme point de départ les pratiques de lecture des enfants et des adolescents dans un contexte de loisii ${ }^{36}{ }$. Ce point de vue paraît cependant isolé. De plus en plus rares en

35. S. Fish, Quand lire c'est faire, ouvr. cité, p. 24.

36. J.-L. Dumortier, Devenir un professionnel de l'enseignement du français..., ouvr. cité, p. IO2-IO3. 
effet sont les didacticiens qui conçoivent la lecture littéraire comme la transposition scolaire d'une lecture savante ou comme un modèle théorique qui ignorerait l'activité du lecteur réel. Au contraire : de même que les premiers promoteurs du «sujet lecteur» ont eu soin de se réclamer du cadre de la «lecture littéraire» (en se référant notamment à Picard), les promoteurs de la conception dialectique de la lecture littéraire ont toujours réservé, quant à eux, une place essentielle au «sujet lecteur».

Il n'en reste pas moins qu'en raison de la nouveauté de leur émergence, la lecture littéraire tout comme le sujet lecteur peuvent faire l'objet, même chez les didacticiens les plus avertis, de malentendus et de flous qui peuvent nuire à leur usage. Pour dépasser ceux-ci, il me semble nécessaire de penser les deux notions ensemble, au sein d'un cadre théorique commun, en considérant la mobilisation de la lecture subjective comme l'une des composantes clés de la lecture littéraire. Le cadre intégrateur de la lecture littéraire constituerait ainsi un garde-fou contre les usages problématiques de la notion de sujet lecteur, et réciproquement, l'importance reconnue au sujet lecteur rappellerait aux promoteurs de la lecture littéraire l'ancrage nécessaire de celle-ci dans une dimension subjective. Qui plus est, la théorie ne doit être qu'un moyen pour mieux agir : lecture littéraire et sujet lecteur doivent donc devenir de plus en plus des notions opératoires, dont l'articulation est rendue visible dans des dispositifs didactiques concrets.

Pour aller dans ce sens, voici un exemple de scénario qui permet d'articuler concrètement la mobilisation du sujet lecteur et la lecture littéraire dans un travail avec les élèves. Ce scénario, qui concerne la lecture de la poésie, comporte quatre étapes dont la chronologie n'a rien de rigide.

La première étape consiste à partir des effets de lecture spontanés des élèves. Après une première lecture du texte, ceux-ci sont invités à répondre aux questions "Qu'est-ce que je vois?", "Qu'est-ce que j'entends?» et "Qu'est-ce que je ressens?». Aux prises avec les images, les sonorités, les rythmes et les effets psychologiques qu'ils éprouvent face au texte, ils mobilisent ainsi en priorité des attitudes de participation émotionnelle et sensorielle qui activent d'emblée une part essentielle de leur subjectivité.

Dans la deuxième étape, il s'agit de construire du sens ensemble. L'élève est ici invité à répondre à la question "Qu' est-ce que je comprends?», en se situant successivement sur le plan thématique (identifier des "champs lexicaux») et sur le plan de la progression (repérer et saisir des syntagmes, des phrases, des strophes - le cas échéant -, puis des unités discursives plus larges). S'adonnant ici à un travail de distanciation et de rationalisation, il ne renonce pas à ses perceptions subjectives, mais il les met pour un temps entre parenthèses pour s'approprier les balises partagées de la communauté interprétative. 
La troisième étape est celle où l'élève est invité à interpréter le texte, c'està-dire à donner un supplément de sens au sens déjà construit. Pour ce faire, il peut se fonder à la fois sur des savoirs communs "externes" au texte (relatifs à la biographie de l'auteur, à l'histoire littéraire, aux intertextes, etc.) et sur des expériences personnelles (liées aux événements de sa vie, à ses rencontres, à ses lectures, etc.) : lecture commune et lecture subjective sont donc appelées ici à se conjuguer de manière explicite.

Vient enfin la quatrième étape, qui consiste à évaluer le texte, c'est-à-dire à porter un jugement sur la portée du sens qui a été compris et interprété. Et, comme pour le sens, les valeurs que l'élève attribuera au texte seront tour à tour subjectives (niveau du jugement de goût, du «j'aime/j'aime pas») et intersubjectives (niveau du jugement de valeur : «ce livre est/ n'est pas important parce que...»), un objectif clé de la formation étant d'apprendre à passer des unes aux autres tout en reconnaissant leur importance à toutes deux.

Ces considérations et cet exemple montrent bien que les malentendus liés aux usages flous des notions de lecture littéraire et de sujet lecteur peuvent être dépassés de deux manières : d'une part par l'arrimage résolu de la notion de "sujet lecteur» à la modélisation dialectique de la lecture littéraire, et d'autre part par des dispositifs didactiques qui attribuent des places égales mais clairement différenciées au "sujet lecteur» et au «lecteur intersubjectif», lequel dépend de l'"autorité des communautés interprétatives» désignées comme telles par Stanley Fish.

Les constats qui précèdent constituent moins des conclusions que des points de départ pour de nouveaux chantiers de recherche en didactique de la littérature. En l'occurrence, trois pistes me sembleraient pouvoir être privilégiées.

En premier lieu, il serait opportun, à mon sens, d'utiliser davantage les notions de lecture littéraire et de sujet lecteur non seulement comme des modèles théoriques, mais aussi comme des outils qui aident à penser l'équilibre de la formation lectorale et littéraire des élèves.

En second lieu, il me semble nécessaire aujourd'hui de stabiliser les notions de lecture littéraire et de sujet lecteur, et cela entraîne deux nécessités : d'une part, confronter les différents modèles qui circulent à propos de ces notions en vue de les clarifier, d'autre part, s'attacher à capitaliser davantage les différentes recherches qui les concernent. Après la prolifération des propositions et des analyses en tous genres, le moment est peut-être venu de rassembler et de tenter des synthèses.

Le troisième et dernier axe consisterait à poursuivre des recherches empiriques, non pas en vue de "valider» à tout prix tel ou tel modèle (ce serait là une visée poppérienne, qui ne me semble avoir de pertinence que pour les 
modèles strictement scientifiques), mais en vue d'analyser ses effets lorsqu'il est mis en œuvre.

À titre d'exemple, il me semble que le modèle de la lecture littéraire peut s'avérer un outil précieux pour décrire les fonctionnements à l'œuvre dans un journal de lecture. Lisons ainsi celui-ci, que Noémie, âgée de I3 ans, a rédigé à propos d'une nouvelle à énigme lue en classe :

J'ai hâte de lire la suite pour voir comment ça va se passer. Arezou croit que c'est une attrape comme les autres textes que Madame nous a donnés. Mike croit que c'est un rêve. Moi je crois que ça s'est vraiment passé et que les «enfants» veulent vraiment la tuer. Je reste sur mon idée et je pense que la suite sera qu'elle voudra prouver son innocence.

On voit là des indices visibles d'un va-et-vient entre l'implication subjective dans le récit et la réflexion distanciée sur celui-ci : intégration des échanges, sélection précise des informations, anticipation sur les relations causales, reformulation du sens global... des caractéristiques qu'il est sans doute possible de repérer sans recourir à un outil théorique, mais auxquelles le modèle de la lecture littéraire et la notion de lecture subjective conferent un supplément de sens et de valeur non négligeable pour l'enseignant comme pour l'élève.

L'objectif de cet article était de contribuer à clarifier et à stabiliser deux notions clés, et par là, d'ancrer un peu plus la didactique de la littérature dans le champ de la didactique du français et des disciplines ${ }^{37}$. Y suis-je parvenu? Tout en l'espérant un peu, je ne me fais pas trop d'illusion, car j'ai bien conscience que, la polysémie des notions et le besoin de singularisation des acteurs étant ce qu'ils sont, cette mise au point ne constituera qu'un jalon au sein du débat dont la lecture littéraire et le sujet lecteur sont l'objet depuis leur origine.

37. Comme on le sait, en effet, les notions et les concepts liés aux différentes didactiques font aujourd'hui l'objet d'un travail d'analyse approfondi, qui parait particulièrement nécessaire. 